that, whatever may be the effect on efficiency at work, noise increases, for example, wastage rate, and number of mistakes.

\section{Physical Acoustics}

Two papers on physical noise problems were presented. Mr. D. M. A. Mercer (University of Southampton) dealt with the difficulties inherent in obtaining accurate measurements of aircraft noise. Such factors as ground reflexions, size and behaviour of source can cause errors of a few decibels ; comparisons of, for example, the performance of jet silencing devices may be quite misleading if these precautions are not taken.

Diesel engine noise was discussed by Dr. A. E. W. Austen and Dr. D. Priede (C.A.V., Ltd.). Speed and swept volume have been related to sound output; there is little variation with load, due to the characteristics of the diesel engine. A quite thorough survey has been made of the vibration of different surfaces of the engine and their effects as noise producers, and sound-levels calculated from these are consistent with measured noise-levels.

\section{Aspects of Noise Analysis}

The second symposium, on "Now Techniques in the Analysis of Noise and Vibration", was held in the Physics Department of the University of Southampton, as a joint meeting with the Institute of Physics. The attendance of about 150, drawn from diverse fields of physics, engineering and electronics, indicated the considerable interest in the analysis of 'noise in the widest sense'. The emphasis was on new and projected techniques; standard bandpass filtering methods received little attention.

The meeting was opened by Mr. R. A. Eades (Signals Research and Development Establishment, Christchurch), who described the speech spectrograph, an instrument capable of examining and analysing short samples of sounds, and presenting the analysis in a manner similar to that of the visible-speech techniques. Mr. Eades made the important point that, in much of this work, bandpass filters with sharp cut-offs are not desirable, due to the 'ringing' they give; the lowered frequency discrimination of a filter of Gaussian or tuned-circuit characteristics is amply repaid by freedom from ringing.

Mr. G. J. Herring (Royal Aircraft Establishment, Farnborough) described an analogue-to-digital con- verter which converted a fluctuating voltage into an input suitable for a Pegasus digital computer. The computer could then be used to make an analysis of the input wave-form. This method was developed because purely analogue methods had proved unsatisfactory. This paper, and the previous one, led to considerable discussion; it was clear that the problem of analysis of random wave-forms was important to many workers. One point, however, was that the distinction between wave-forms with and without a periodic component-a difference important to the statistician -was not always made clear; with consequences perhups of unnecessary difficulties in many analyses.

Mr. D. M. A. Mercer (University of Southampton) emphasized the practical point that, in any analysis, frequency discrimination, stability of estimates and ease of computing were all linked, and could not all be maximized at once; great care was needed in the planning stages of an analysis. Cross-correlation techniques were often able to give results unobtainable by straightforward methods.

Dr. G. M. Jenkins (Imperial College of Science and Technology, London) discussed the statistical implications of obtaining a spectrum via an autocorrelogram. It is desirable to examine the autocorrelogram itself before the Fourier transform process; in any event, some modification to it is often desirable, to obtain a more stable spectrum, but in addition much information is often available at this stage; and different applicable statistical criteria were described.

The ensuing discussion demonstrated much interest in the use of axis-crossings of a random function. Presumably there is a major theoretical breakthrough to be looked for on this topic ; but apart from this, theoretical and experimental studies demonstrate that axis-crossings carry most of the information of a wave-form (apart from magnitude, of course). The limitations of the method, however, are not yet known.

The concluding impression of the symposium appeared to be twofold: first, that 'noise in the widest sense' is a subject of considerable interest, and secondly, that there often appeared to be a considerable gap between the theoretical studies, and the work of those concerned with practical noise and vibration analysis. This leads, at best, to correct analyses obtained with unnecessary difficulty, and at worst, analyses which are meaningless. Closing of this gap would be of benefit to all concerned.

Derwent M. A. Mercer

\title{
BIOSYNTHESIS AND SECRETION OF ADRENOCORTICAL STEROIDS
}

T a symposium held on February 14, the Bio-
chemical Society gathered together a group of
investigators who described their own work and that
of others on various aspects of the biogenesis of the
adrenocortical hormones. In an introductory paper,
I. E. Bush described the chemical nature of the
steroids under discussion and the applieation of
various types of chromatographic procedures to the
separation and identification of these substances. An
important feature of steroid chromatography is the
very large number of possible compounds and the
danger of wrong identification if chromatographic
properties are relied upon to the exclusion of others.
This danger may be diminished by the use of numerous microchemical and spectrophotometric techniques. The former include acetylation or oxidation (notably the removal of the C-17 side chain) on the 10-20 $\mu \mathrm{gm}$. scale, followed by observation of the chromatographic behaviour of the product on paper. Spots of steroid on paper give various well-known colour and very sensitive fluorimetric reactions. On the physical side a wide range of correlations between structure and absorption bands in the infra-red region has been established. Ultra-violet absorption spectra in sulphuric acid and alcoholic alkali provide useful information which, in conjunction with paper chromatography and comparison with reference substances, may provide identifications almost as reliable 
as those from infra-red spectra. New work in Bush's laboratory has shown that useful generalizations about the behaviour of steroids in partition systems can be made using Martin's theory and the $R_{M}$ function of Bate-Smith and Westall. For this theoretical approach Bush has collected numerous $R_{F}$ values from the literature. Not all will agree with him on the reliability of these values. The chromatogram tank is not yet a precision instrument.

J. K. Grant then reviewed present knowledge of the biosynthesis of adrenocortical steroids. Here there are at least three questions to be answered. What is the nature of the secretion of the adrenal cortex, what sequences of reactions are involved in the syntheses of the steroids in the cortical cells and what are the mechanisms of these reactions? Complete answers cannot be given. The analysis of adrenal venous blood collected from animals and from human subjects undergoing adrenalectomy has provided direct evidence of the nature of the steroids secreted by the adrenal gland. Hydrocortisone, corticosterone and aldosterone are the principal $\mathrm{C}_{21}$ compounds found. The last of these, and a variety of others of less physiological importance, are present in trace amounts. There is indirect evidence that the adrenal cortex in man secretes a substance which, in contrast with aldosterone, promotes the excretion

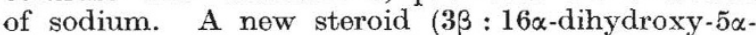
pregnan-20-one), which produces this effect in rats, has recently been isolated by Wettstein from the adrenal glands of swine. There is also evidence that the adrenal gland secretes androgenic and ostrogenie steroids.

Application of a variety of techniques has clearly established that adrenocortical steroids may be formed in the gland from acetate or cholesterol by formation and subsequent hydroxylation of progesterone. An outstanding question of major interest is the possibility of alternative pathways to those originally proposed. These alternatives are concerned with the order in which the hydroxylations of the key intermediate progesterone proceed, with the possibility that cholesterol is not an obligatory intermediate in hormone formation, and with the direct transformation of cholesterol to $\mathrm{C}_{19}$ steroids. Hydroxylations commonly oceur at C-17, C-21 and $\mathrm{C}-11 \beta$ in that order. It has been shown beyond reasonable doubt that a C-21 hydroxy steroid cannot undergo hydroxylation at C-17. Grant has, however, demonstrated that $11 \beta$-hydroxylation of progesterone can proceed in good yield. Hydrocortisone may then be formed from 113-hydroxyprogesterone but at rates too slow to be of significance in normal biosynthesis. This alternative pathway may, however, be important in pathological states. Despite much suggestive evidence and numerous experiments with isotopically labelled compounds, it has not been possible to establish firmly the existence of alternative pathways from acetate to adrenocortical hormones which do not involve cholesterol. When such pathways appear to have been demonstrated, alternative explanations of the results have been possible if the existence of different metabolic pools of adrenal cholesterol is assumed. In other cases the results are unacceptable since the comparisons between the specific activities of precursors and products have been made at one point in time and not on a kinetic basis known to be essential in order to avoid misinterpretation of results. Despite intensive study, the mechanism of steroid hydroxylation has not been elucidated. Molecular oxygen and reduced triphosphopyridine nucleotide are essential. It has not been possible to confirm that a transhydrogenase is involved in the formation of reduced triphosphopyridine nucleotide. It has been suggested that the reduced nucleotide is required for the reduction of an oxygen activating or transferring enzyme containing a heavy metal, but proof is lacking. There are, however, indications that the 11ß-hydroxylating enzyme system located in the mitochondria is complex. The observation that 2-methyl-1:2-bis-(3pyridyl)-1-propanone (SU.4885) appears to be a specific inhibitor of $11 \beta$-hydroxylation in vivo and in vitro has aroused considerable interest.

Adrenal glands of the rat, ox and man have been most extensively studied. The anatomy and histology of these glands were described by T. Symington. He directed attention to two features which have not until recently received adequate attention from biochemists : the unusual musculature of the adrenal veins which may, by restricting blood flow through the gland, influence steroid biosynthesis, and the marked differences in histology between the ruminant and non-ruminant adrenal cortex. The fascicularis and reticularis zones, readily distinguished in nonruminants, are not distinguished in the ruminant. In the ox, which is typical of the ruminants, a broad zone of 'compact' cells fills the cortex from the medulla to the prominent glomerulosa beneath the capsule. In man, the fasciculata zone of variable width is filled with lipid-laden 'clear' cells and lies between the reticularis zone of 'compact' cells and the glomerulosa, which is very irregular, forming islands under the capsule so that glomerulosa cells are absent from some sections. The ox adrenal gland is for this reason a more satisfactory object than the human gland for the in vitro study of the synthesis of aldosterone which appears to occur in glomerulosa cells. The morphology and histochemistry of 'clear' and 'compact' cells and the influence of corticotrophin thereon have been studied in Symington's laboratory using glands removed surgically in two stages for the treatment of breast cancer. The use of these fresh glands has also permitted the study of enzyme reactions involved in steroid biosynthesis. These biochemical studies and in addition the results of analysis of adrenal venous blood obtained at the time of adrenalectomy have been correlated with the morphological and histochemical observations. It would appear from the results obtained that 'clear' cells of the fasciculata zone may function as stores of hormone precursor, and that the 'compact' cells of the reticularis zone, far from being the senescent cells described by others, may be the site of active hormone biosynthesis. 'Clear' cells are converted to 'compact' cells under the influence of corticotrophin, which stimulates corticosteroid secretion, influencing various steps in the sequence of biosynthetic reactions. Studies with the electron microscope in Glasgow have revealed important differences between the 'clear' and 'compact' cells, the most striking features of which are the mierovilli, which form extensions of the cell membrane running out into the intercellular spaces. The function of the microvilli is unknown, but it is interesting to speculate that they may be concerned with secretory processes.

P. J. Ayres described the relation of steroid secretion to the histological zones of the adrenal cortex, with particular reference to the evidence which he has obtained in collaboration with Dr. and Mrs. Tait that aldosterone is synthesized and secreted 
by the cells of the glomerulosa zone. This work developed out of early observations on the rat ; these supported the view that a steroid active in electrolyte metabolism was secreted by the glomerulosa zone, relatively independent of the pituitary but influenced by electrolyte intake. Further indirect evidence followed the isolation of the steroid concerned, aldosterone, from whole adrenal extract by the Taits in 1952. It was possible to show that aldosterone secretion, but not that of corticosterone, is diminished in rats on a low-potassium diet. Hypophysectomy has a much more pronounced effect on corticosterone than on aldosterone secretion in the rat. After this operation in dogs, the fasciculata-reticularis zone of the adrenal gland atrophies and the secretion of hydrocortisone and corticosterone falls ; by contrast the glomerulosa zone and the secretion of aldosterone are unaffected. Direct evidence for the preferential production of aldosterone in the glomerulosa zone was obtained by the Taits and their collaborators by incubation of adrenal capsule strippings, to which glomerulosa cells were adhering, and of tissue from the underlying zone. In the ox the 17-hydroxylating enzyme system appears to be in the zone lying under the glomerulosa only, whereas the 18-oxidase system necessary for aldosterone synthesis is confined to the glomerulosa zone.

A remarkable feature of the adrenal cortex is its ability to synthesize all known types of steroid hormones. R. V. Short discussed the biosynthesis and secretion of sex hormones by the adrenal gland. In his laboratory, methods have been developed for the separation and determination of the major and some of the minor steroid constituents of adrenal venous blood. These have been applied to the analyses of blood collected from human subjects by A. P. M. Forrest in Glasgow, and from adult, newborn and fotal domestic animals by Dr. Short's collaborators in Cambridge. Progesterone has been found in adrenal blood from women, cows, sheep and pigs in concentrations higher than in the peripheral blood. 17 $\alpha$-Hydroxyprogesterone, a steroid which is sixty times as active as progesterone in some biological assays, has been detected in the adrenal venous blood of women and cows. The interesting observation has been made that young calves secrete 20 $\alpha$-hydroxypregn-4-en-3-one. This disappears from the secretion some time after birth and is replaced by the 20ß-epimer in the adult animal. The significance of this is unknown. There is good indirect evidence for the secretion of œestrogens by the adrenal cortex, but they must be present in human adrenal venous blood in amounts too small for detection by present methods. Four androgens have been reported in adrenal-vein blood in man. Dehydroepiandrosterone is probably exclusively of adrenal origin, but its precursors are still uncertain. Androstenedione is undoubtedly the most biologically active adrenal androgen and it may be present in adrenal venous blood in relatively high concentration. It is remarkable that androstenedione and dehydroepiandrosterone cannot be detected in even large samples of adrenal venous blood in cattle. 11ß-Hydroxyandrostenedione is probably present in the adrenal venous blood of the cow and sheep as well as in that of the rat and cat, but this compound, in common with the other known 11-oxy $\mathrm{C}_{19}$ steroids, seems to possess little biological activity. These observations may be of considerable importance in veterinary medicine and cast doubts on the reports of adrenal virilism in cattle.
The amount of corticosterone and hydrocortisone secreted by the adrenal cortex is controlled by corticotrophin from the anterior lobe of the pituitary. How the pituitary obtains the information necessary to enable it to adjust the activity of the adrenal cortex to the body's requirements is a fascinating question which has recently attracted much attention. Knowledge of this matter was reviewed by Marthe Vogt. Few histologists consider the nerve supply to the adenohypophysis adequate for the control of corticotrophin secretion, and this control is therefore assumed to be humoral. It has been shown that substances such as adrenaline, which may be carried by the systemic blood, can promote the release of corticotrophin by the pituitary. There is also much evidence in support of the view that a stressing stimulus results in nervous activity in the hypothalamus which elaborates a 'corticotrophin-releasing factor'. This is carried by a 'portal' circulation to the anterior lobe, where it stimulates corticotrophin secretion. The original suggestion that the corticotrophin-releasing factor is identical with vasopressin has been modified by the independent observations of Saffran and Guillemin, who now propose that it is a polypeptide similar to but different from vasopressin. The purified material is active in vitro in nanogram amounts, and microgram quantities produce a similar effect on the rat pituitary stores of corticotrophin or on blood corticosteroids as severe stress. The active material contains seven of the eight amino-acids of lysine-vasopressin and, in addition, serine and histidine. Dr. Vogt also discussed the nature of stimuli which activate or inhibit hypothalamic activity. The level of corticosteroids in blood is now regarded as a contributing, but not the sole, factor accounting for the control of corticotrophin secretion in stress. Recent work suggests that the suppressing effect of high doses of corticosteroids may be on the hypothalamus rather than on the pituitary. In addition to lack of corticosteroid in the blood, afferent nervous impulses, especially if they elicit pain or worry, stimulate the release of corticotrophin, but the nature of the stimulus or stimuli which act in other forms of stress is unknown. General depressants of the brain inhibit the release of corticotrophin.

Dr. Vogt also reviewed knowledge of the control of secretion of aldosterone. This secretion is less influenced by corticotrophin than that of the glucocorticoids. Farrell has, however, recently found that certain preparations of eorticotrophin increased aldosterone secretion in the decerebrate, hypophysectomized dog, but satisfactory evidence for the existence of a specific pituitary hormone which influences aldosterone secretion is lacking. The role of such a hormone would be limited, since increased aldosterone secretion in the hypophysectomized dog has been obtained with a variety of stimuli. Experiments in Dr. Vogt's laboratory have demonstrated the rapid and reversible response of aldosterone secretion in the dog to expansion and contraction of the intravascular volume. Infusion of blood decreases, whereas hæmorrhage increases, the secretion of aldosterone. The former effect was less easily elicited than the latter.

In the final paper, N. Saba dealt with the mode of action of corticotrophin from the biochemical point of view. He referred to the observations of Hechter that corticotrophin acts on the conversion of cholesterol to pregnenolone and the later independent work of Heard and Grant on the stimulation of 
11-hydroxylation by the trophic hormone. Saba's results obtained in collaboration with Hechter suggest that corticotrophin influences the spatial relationships of enzymes involved in the biosynthesis of adrenocortical steroids. More recent work by others has revealed an effect of corticotrophin on enzyme systems which effects the reduction of triphospho- pyridine nucleotide. The requirement for reduced triphosphopyridine nucleotide for steroid hydroxylation has already been referred to. If this is an important effect of corticotrophin it is difficult to relate it to the specificity of action of this hormone.

J. K. Grant

\section{EDUCATION IN THE UNITED STATES (1957-58)}

$\mathrm{E}^{\mathrm{n}}$ DUCATION in the United States of America is based on three fundamental concepts: that the primary responsibility for public education rests with the States; that every person has an equal right to educational opportunities ; and that educated citizens are essential to freedom and human welfare. Working within these concepts during 1957--58 the United States made further progress towards its goal of improving education for all*.

Evidence of progress is found in the increasing educational attainment of the population. The number of school years completed by the average adult 25 years of age and over increased from $9 \cdot 3$ in 1950 to 10.6 years in 1957 . The group 25-29 vears had completed $12 \cdot 3$ years of schooling, while the group older than 65 had completed only $8 \cdot 3$ years.

Although education is a State responsibility, no State administers its schools directly. Laws have been enacted in each State dividing the territory of the State into local school administrative units, commonly termed school districts. The powers of local district school boards to establish and maintain schools are prescribed by State law, but permit exercise of local initiative in exceeding minimum educational standards required by the State. Each State has its own department of education, which exercises controls and provides specialized services to assist local school districts in conducting the State programme of education.

At the beginning of the school year there were $1,152,500$ instruction rooms in full-time public elementary and secondary schools; an additional 142,300 rooms were needed to relieve over-crowding and to replace unsatisfactory facilities. About 70,500 instruction rooms were scheduled to be built during the year. About 61,000 rooms will be needed to accommodate next year's enrolment increase and to replace rooms that will probably be abandoned.

Almost one-third of all pupils enrolled in the public elementary and secondary schools are transported to and from school at public expense. During $1955-56$, more than 10 million pupils were transported. In most States pupils must live $1 \frac{1}{2}$ miles or more from the school to be eligible for transportation for which the State helps to pay the cost.

Institutions of higher education classified by type of support and control are of two general typespublicly controlled and privately controlled. Onethird of the approximately 1,900 higher institutions are publicly controlled and supported by public or government agencies; two-thirds are privately controlled and supported by individuals or ecclesiastic, philanthropic and other groups. The State exercises little control over institutions of higher educa-

* Progress of Public Education in the U.S.A., 1957-58. (Washington, D.C. : Gov. Printing Office; available also from H.M. Stationery Office, London.) tion, even those supported by public funds, and consequently both types of institution operate with a high degree of autonomy.

In 1957-58 expenditures for education in public elementary and secondary schools and in higher institutions totalled 20,000 million dollars, which was $5 \cdot 5$ per cent of the 1957 total national income of 358,000 million dollars. Funds to cover expenditure in public schools were provided by Federal, State and local governments.

For the fiscal year 1958 the Federal Government appropriated approximately 2,000 million dollars for educational purposes. The total included funds administered by the Office of Education and was distributed as follows : Office of Education, 7,000,000 dollars; vocational education, 40,888,412 dollars; higher education, 5,051,000 dollars; school construetion and maintenance, 225,650,000 dollars; and library services, $5,000,000$ dollars.

Since the States have primary responsibility for public education in the elementary and secondary schools they provide funds and authorize local school districts to provide local tax-funds for public schools. In the 1957-58 school year it is estimated that of the total revenue for public schools the Federal Government provided 4 per cent; the States, 41 per cent; and local districts, 55 per cent. In recent years the percentage from Federal and States funds has been increasing slightly.

As much as $\mathbf{9 3}$ per cent of local educational revenue is obtained from property taxes. Local communities use the property tax to secure funds for current operating expenses and for school construction. Some local districts also levy non-property taxes for schools, including local per capita taxes and taxes on wages, sales and amusements. Non-property taxes for schools produce 7 per cent of local revenue.

Total expenditure per pupil in 1957-58 averaged 431 dollars, an increase of about 7 per cent over the amount in $1955-56$.

Tax revenues supply most of the funds for public institutions; private donations, student tuition and endowment supply most of the funds for private institutions. The 1957 budgets for both private and public institutions for educational and general expenditures, excluding auxiliary services, student aid and plant expansion, totalled 3,200 million dollars. Of this, students paid 950 million dollars as tuition ; earnings on endowment provided 150 million dollars ; private gifts, 250 million dollars.

The organization of public schools is determined by State and local authorities, but generally the basic 12-year programme is organized as an 8-year elementary and a 4-year secondary programme or a 6-year elementary and a 6-year secondary programme. Typically a 6-year secondary programme is divided into a 3 -year junior and a 3 -year senior high school. The most common type of school is one 